\title{
Fractional-Order Identification Based On Time Domain Methodology For Hydraulic Canal System
}

\author{
Gharab Saddam ${ }^{1}$ \\ Vicente Feliu-Batlle ${ }^{1}$ \\ Juan Carlos Cambera ${ }^{1,2}$ \\ Saddam.Gharab@alu.uclm.es \\ Vicente.Feliu@uclm.es \\ JuanCarlos.Cambera@alu.uclm.es \\ ${ }^{1}$ Escuela Tecnica Superior de Ingenieros Industriales Ciudad Real, Spain \\ ${ }^{2}$ Instituto de Investigaciones Energeticas y Aplicaciones Industriales Ciudad Real, Spain
}

\begin{abstract}
This paper discusses the identification of hydraulic canal system with a fractional-order model. The identification procedure is carried out by using a time domain technique. A pseudorandom binary signal (PRBS) is considered, the auto-correlation of the input signal, as well as, the impulsive response of the system has been concluded, and the obtained model are discussed in order to show the effect of using the PRBS signal in comparison with a fundamental step signal. Finally, we put the accent into the improvisation of the fractional-order model of the considered prototype that has a significant impact on improving the performance of canal monitoring, control, and supervisory systems.
\end{abstract}

Keywords: Fractional-order model, fractionalorder identification, PRBS, Hydraulic canal system.

\section{INTRODUCTION}

Although its application has shown interest only in recent years, the theory of fractional-order calculus has quite a long and prominent history. It has defined as the generalization of classical calculus to orders of integration and differentiation not necessarily integer, and it has represented a matter of almost exclusive interest for few mathematicians and theoretical physicists.

The complexity of such calculus, the evident selfsufficiency of the integer order calculus, and the fact that it does not have a fully acceptable geometrical or physical interpretation are the reasons of not being widely used in engineering sciences [10]. During the last decades, it has been presented as an efficient tool that models more precisely some physical engineering processes, and accordingly it was used as a promissory tool in bioengineering [11], electronics [1], viscoelasticity [5], robotics [6, $7]$, control theory [2, 4], and signal processing [12, 19] and others.

Thereafter, system identification using fractional order models still represents a complicated task in comparison with classical identification. In the case of classical integer systems, once the order of the system to be identified is selected, the parameters of the model can be optimized directly. Although, for fractional-order systems, identification procedure requires the choice of the number of fractional operators and the fractional power of these operators [14].

Thus, the identification process is significantly complicated each time the integer order is lost. Previous work in this area has been limited [3, 8, 18]. The authors in the before mentioned works identify mechanical damping behavior and an electrode-electrolyte polarization for specifically chosen transfer function forms and by using frequency domain techniques. Fractional order differential equations are more adequate than integer-order equations on modelling diffusion systems as occurs in electrochemical processes [16, $17]$.

Works published in [13, 15] shown that there is great potential for the use of fractional-order models in depicting the dynamic behavior of irrigation main canal pools. According to the same references, fractional-order models could accurately represent the dynamic model of hydraulic canal systems, which has a significant impact on improving the performance of canal monitoring, control, and supervisory systems. 
In this work, fractional-order model for hydraulic canal system is calculated using a new identification technique based on time domain data. A new model has been explained, for the considered system, in order to enhance the precision of the obtained results. The identification procedure is based on PRBS signal which has been generated in function of the parameter of the system. The impulsive response of the system is calculated from the cross-correlation function of the resulting signals, and the fractional-order transfer function is concluded using the Laplace transform.

The paper is organized as follows, the second Section represents a small definition of the considered system, Section 3 represents the new model of the considered SISO system, In Section 4, a comparison between the models obtained using the PRBS signal as an input on time domain identification and the classical identification based on step input signal is developed, and finally, Sections 5 represents some conclusions.

\section{THE HYDRAULIC CANAL PROTOTYPE}

As it was mentioned in the introduction, the considered system is a closed-loop water variable slope rectangular canal characterized by glass walls and a methacrylate bottom and located in the Fluids Mechanics Laboratory in the University CastillaLa Mancha (Spain). The description of the canal is like $5 \mathrm{~m}$ of length, $8 \mathrm{~cm}$ of wide, and $25 \mathrm{~cm}$ high for the walls. The platform of the prototype integrates electromechanical sensors and actuators, a PLC (programmable logic controller) and a SCADA (data acquisition and supervisory system). The actual configuration is composed of two pools: the first one acting basically as a reservoir and the second one acting as the main canal pool of approximately $4.7 \mathrm{~m}$ in length with a downstream end operation (delivers the required water flow at its downstream end).

The upstream gate of the main canal pool is a motorized undershot gate which connects the two pools, and the downstream gate is a manually adjustable overshot gate. The general view of the prototype is presented in Figure 1, and another schematic representation is depicted in Figure 2.

The geometry of the downstream overshot gate is shown on the left-hand side of Figure 2. The angular position of this gate can be manually adjusted to three values which yield gate top heights of 13 , 23 and $33 \mathrm{~mm}$ above the canal bottom respectively.

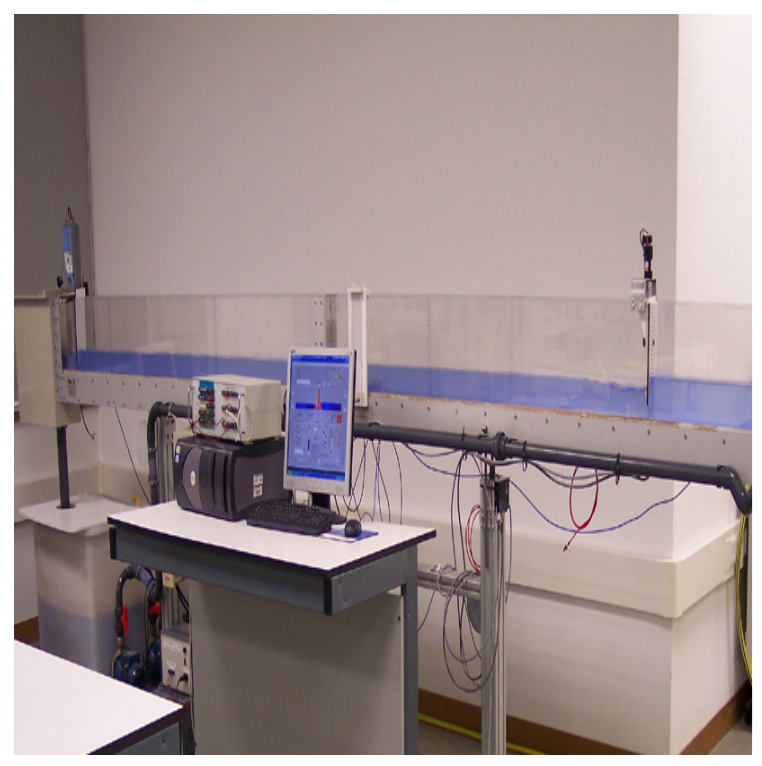

Figure 1: Prototype hydraulic canal in laboratory

\section{Time domain Identification}

\subsection{Fractional-order model for the hydraulic canal prototype}

The dynamics of the hydraulic canal are described by the Saint-Venant equations, which are nonlinear hyperbolic partial differential equations [8]. Linearized models around some flow regimes are often used in order to design canal controllers. The parameters of these approximated LTI models change depending on the operation regime of the canal. Consequently, experiments based on the responses to step inputs of the considered system were carried in the aim to obtain linear dynamic models that describe its dynamic behavior around several flow regimes. Transfer function in equation (1) represents the optimal model precisely define the dynamic of the hydraulic canal system where the time delay $L=4.95$, the time constant $T=1.82$, the static gain $K=0.273$, and the fractional-order characterizing the system is 


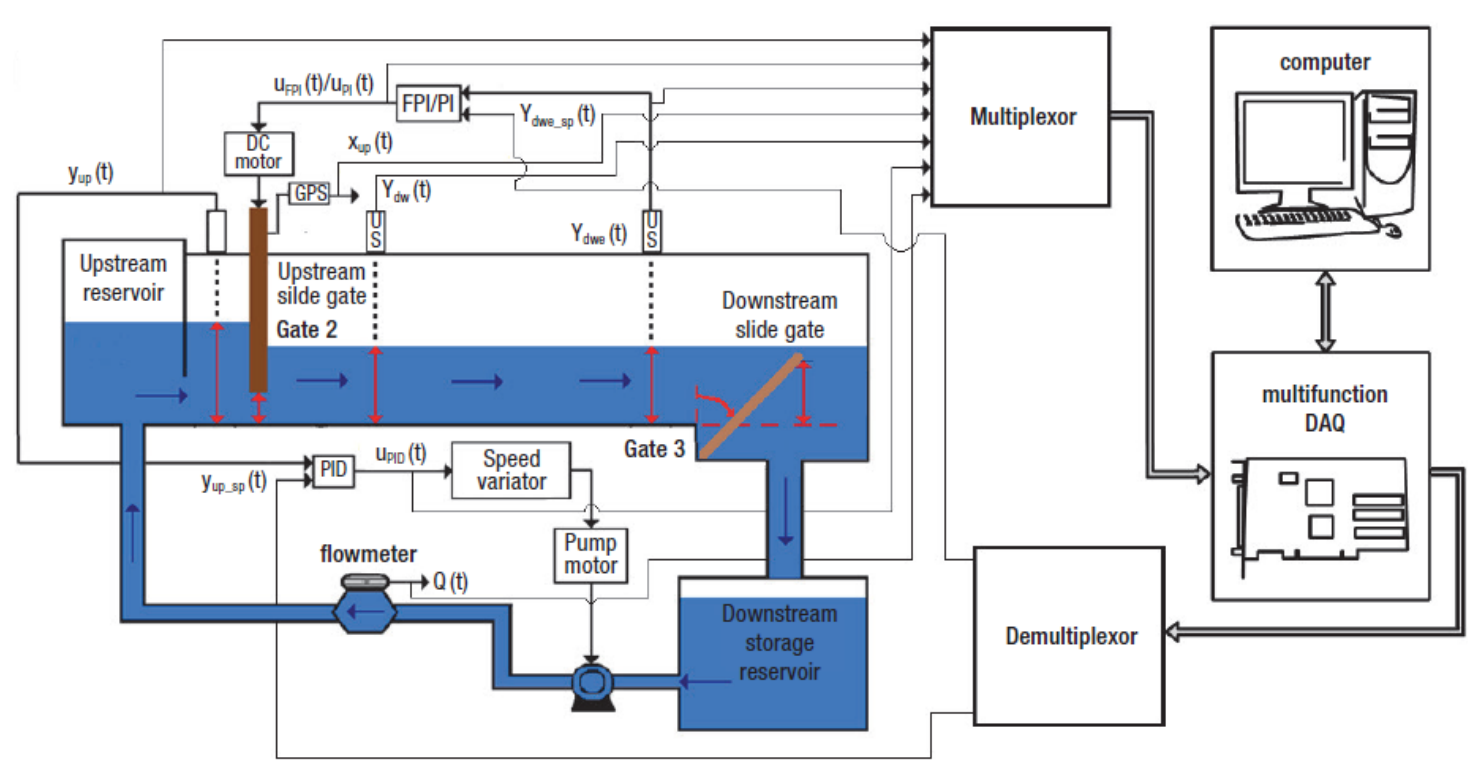

Figure 2: Schematic representation of the prototype hydraulic canal

$\lambda=0.85$.

$$
G(s)=\frac{K}{1+T s^{\lambda}} e^{-L s}
$$

\subsection{Characteristics and design of PRBS}

A Pseudorandom binary signal (PRBS) finds applications over many disciplines for system identification. The frequency spectrum of the PRBS is known as an approximation of the limited bandwidth of the white noise, and then it represents a useful stimulus for frequency response analysis. By definition, the PRBS consists of a random sequence of binary states that usually generated by means of a shift register with feedback paths. The size of the sequence (M) depends on the number of bits $(\mathrm{N})$ of the shift register and the positions of the feedback paths.

\subsubsection{PRBS generation}

The PRBS signal of Figure 3 has been designed according to the following system constraints:

- The frequency of the PRBS signal is fixed to cover the bandwidth of the considered system. In the case of our model, the time constant is $T=1.82 \mathrm{~s}$, the bandwidth that covers the system frequencies is $B W=5.4 \mathrm{~Hz}$ and then, the period of the PRBS is $T_{P R B S}=1.2 \mathrm{~s}$.

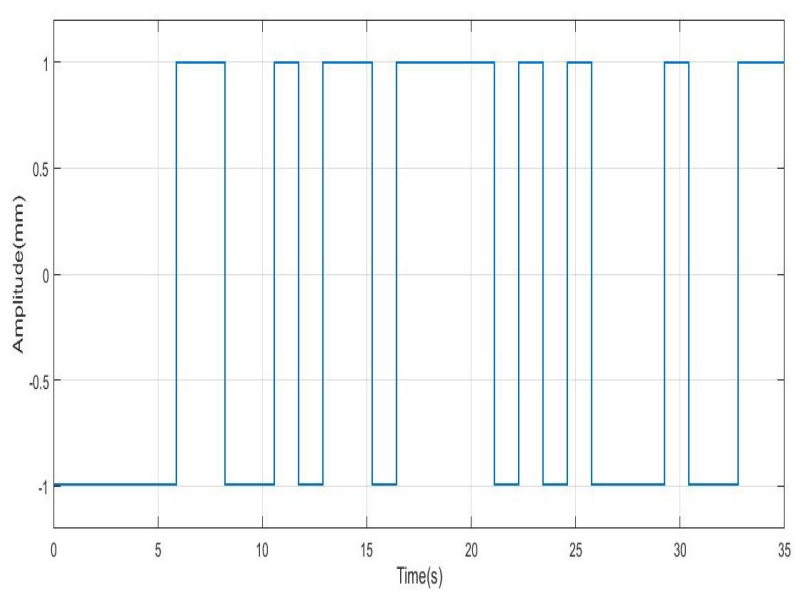

Figure 3: PRBS signal designed according to the system constraints

- The time duration of the PRBS signal can be defined as, the shorter necessary time needed by the impulsive response, of such system, to reach and stabilize into the zero value. This theoretical value can be assured according to equation :

$$
D_{P R B S}>3 T+L
$$

In the case of the hydraulic canal system, we are taking into account the time delay of the system approximated by $\mathrm{L}=4.95 \mathrm{~s}$, the delay caused by the gate translations each time a new reference is applied to the system. Also, 
we are considering the sampling time of the system.

In order to precisely reproduce the impulsive response of the considered model, the number of samples per period for the PRBS signal has to be enough. Accordingly, an additional time has been added to the periodicity of the PRBS signal and the new time duration of the PRBS signal is now: $D=15 s$. The number of bits associated with the periodicity of the PRBS signal is concluded from equation (3).

$$
2^{n-1}=D_{P R B S}, n=4.90 ;
$$

The number of bits has been concluded then equal to 5, and the length of the PRBS signal corresponding is $D=31 \mathrm{~s}$

- The sampling time of the system is $h=0.13 s$.

- The amplitude of the PRBS signal equal to $1 \mathrm{~mm}$.

\subsubsection{Gate model}

In order to achieve the time domain identification by using the PRBS signal, the upstream gate dynamics were analyzed, and a new gate model has been developed and showed in equation (4), where the aim is to improve the identification performances. In that equation, $Y_{G}^{*}$ represents the gate reference, $Y_{G}$ represents the actual position of the gate, $\dot{Y}_{G}$ represents the derivative of the gate position, and $v$ represents the velocity of the gate.

$$
\dot{Y}_{G}= \begin{cases}v \operatorname{sign}\left(Y_{G}^{*}-Y_{G}\right), & \text { if } Y_{G}^{*} \neq Y_{G} \\ 0, & \text { if } Y_{G}^{*}=Y_{G}\end{cases}
$$

These dynamics could affect the linearity of the system, certainly that the period of the PRBS signal is very small compared to the final time of a step signal. The new signal that reaches the model of the hydraulic canal system is detailed in Figure 4 in red color, the injected PRBS signal represented in blue color to put the accent into the real function of the Gate model, how much the reference signal can be affected by that model and by the way the improvement of the final model for the considered system.

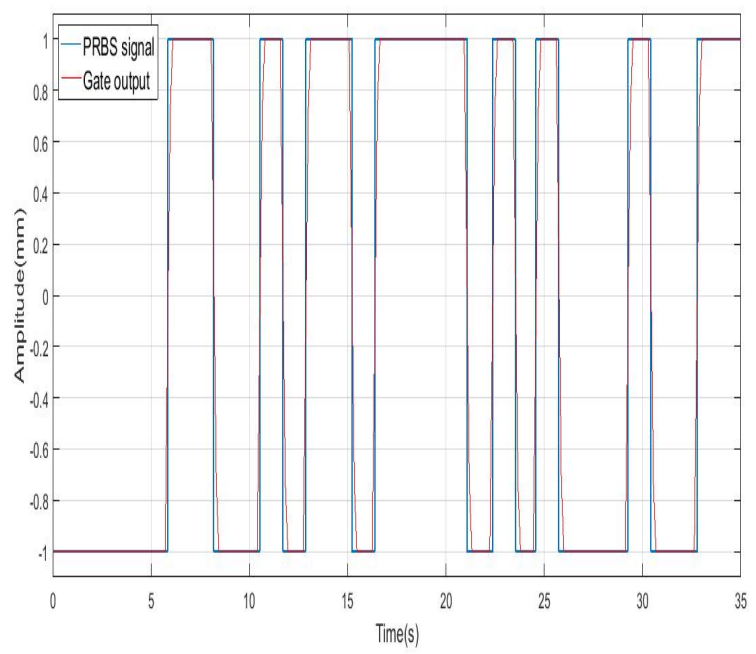

Figure 4: PRBS signal affected by the gate model

\subsubsection{Quantization model}

The sensors of the hydraulic canal system characterised by a resolution factor equal to $0.05 \mathrm{~mm}$. Accordingly, a quantizer block is used to model this resolution and the obtained signal will be modified according to the following equation where $y_{s}$ represents the output of the system, $y_{q}$ represents the output of the quantization model , $r=0.05$ represents the resolution of the sensors, the nearest integer function is defined such that $\operatorname{nint}(x)$ is the integer closest to $x$.

$$
y_{q}=r * \operatorname{nint}\left(\frac{y_{s}}{r}\right)
$$

\section{Simulation results}

In this section, simulations results have been obtained using the identification procedure described in this work and the models of the fractional-order model of the hydraulic canal system, the model of the upstream gate, and the resolution of the sensor developed on Simulink Matlab.

First, the PRBS signal already defined in the previous part is used as an input signal, the gate model affect the input according to Figure 4, and the output signal is quantized with an order corresponding to the resolution of the sensor. The impulsive response of the system has been concluded from the cross-correlation function of the two sig- 
nals (input and output). The obtained fractionalorder model appears in table 1 in the first position and in order judge the precision of the new approximated model, time responses are compared with that corresponding to the reference model in Figure 5. In addition, The performance is determined with the Normalized Root Mean Square Error (NRMSE) defined by the equation (6), where $y_{r}$ is the response of the reference model and $y_{a p p}$ is the response of the identified model for the same input.

$$
N R M S E=100\left(1-\frac{\left\|y_{r}-y_{a p p}\right\|}{\left\|y_{r}-\operatorname{mean}\left(y_{r}\right)\right\|}\right)
$$

In the second case, the input is a step signal of an amplitude equal to $2 \mathrm{~mm}$ and a final time equal to $40 \mathrm{~s}$. In both cases, the output signals have been saved.
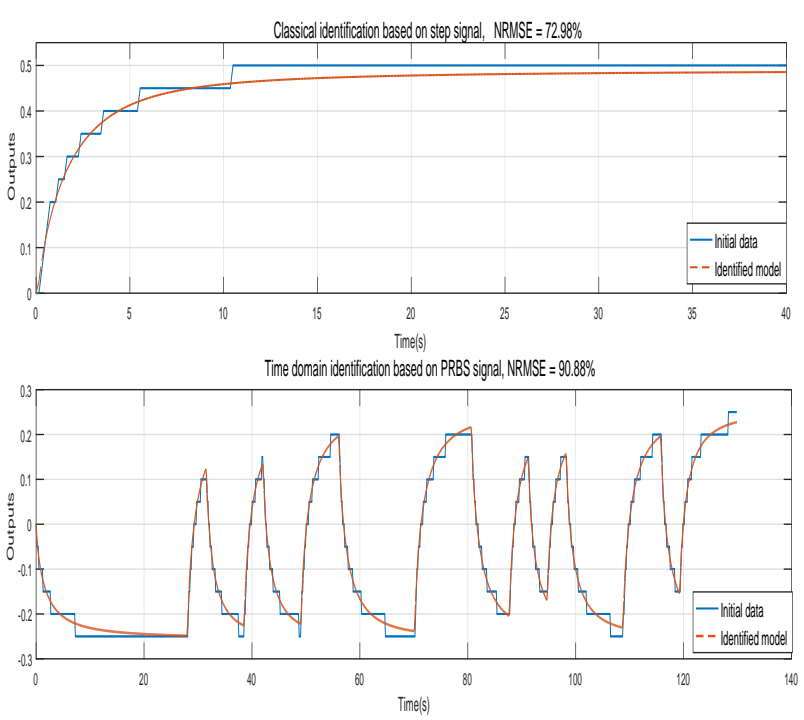

Figure 5: Fractional-order Identification based on PRBS and step signals for the new system model

Table 1: Model parameters obtained by time domain identification in comparison with the classic identification based on step signal

\begin{tabular}{|l|l|l|l|l|l|}
\hline Input signal & $K_{f}$ & $L_{f}$ & $T_{f}$ & $\lambda$ & NRMSE \\
\hline PRBS & 0.26 & 4.95 & 1.72 & 0.76 & $90.88 \%$ \\
\hline Step & 0.246 & 4.95 & 1.73 & 0.84 & $72.98 \%$ \\
\hline
\end{tabular}

\section{Conclusions}

This article has addressed the identification of the laboratory hydraulic canal model by a delayed fractional-order transfer function. The elaborated identification procedure was based on time domain data, PRBS signal has been considered according to the model parameters, the new improvements to the system in relation with the real components of our hydraulic canal, and the sampling time of the system. A new model has been developed for the upstream gate in order to read precisely the references data injected to the system, and another modelization has been carried out in accordance to the sensor's resolution in order to carefully measure the output obtained signal.

Using the output signal to an input of form PRBS, the impulsive response of the system is concluded from the cross-correlation of the two signals, the transfer function is obtained, and the identification procedure has been discussed in comparison with another identification carried out by a step signal.

In order to prove the added value and the benefits from using a PRBS input signal on system identification procedure, a new aleatory signal was used to justify the precision of the obtained model and accordingly, the normalized root mean square error confirm in percent how much improve this technic the model of the hydraulic canal.

\section{Acknowledgement}

NO acknowledgements.

\section{References}

[1] B. T. Krishna and K. V. V. S. Reddy, (2008) Active and passive realization of fractance device of order, Active and Passive Electronic Components, Article ID 369421, 5 pages.

[2] G. W. Bohannan,(2008) Analog fractional order controller in temperature and motor control applications, Journal of Vibration and Control, vol. 14, pp 1487-1498.

[3] H. H. Sun, B. Onaral, Y. Tsao,(1984) Application of positive reality principle to metal electrode linear polarization phenomena, IEEE 
Trans. Biomed. Engrg. BME-31 (10) pp 664674.

[4] J. Cervera and A. BaËœnos,(2008) Automatic loop shaping in QFT using CRONE structures, Journal of Vibration and Control, vol. 14, pp 1513-1529.

[5] J. De Espindola, C. Bavastri, and E. De Oliveira Lopes, (2008) Design of optimum systems of viscoelastic vibration absorbers for a given material based on the fractional calculus model, Journal of Vibration and Control, vol. 14, pp 1607-1630.

[6] J. Rosario, D. Dumur, and J. T. Machado, (2006) Analysis of fractional-order robot axis dynamics, in Proceedings of the 2nd IFAC Workshop on Fractional Differentiation and Its Applications, vol. 2.

[7] M. F. M. Lima, J. A. T. Machado, and M. Crisostomo, (2007) Experimental signal analysis of robot impacts in a fractional calculus perspective, Journal of Advanced Computational Intelligence and Intelligent Informatics, vol. 11, pp. 1079-1085.

[8] M. H. Chaudhry, (2008) Open-channel flow, 2nd ed. New York: Springer.

[9] N. M. M. Maia, J. M. M. Silva, A. M. R. Ribeiro, (1998) On a general model for damping, J. Sound Vib. 218 (5), pp. 749-767.

[10] R. E. Gutierrez, J. M. Rosario, and J. T. Machado,(2010) Fractional Order Calculus: Basic Concepts and Engineering Applications, Mathematical Problems in Engineering, Article ID 375858, 19 pages.

[11] R. L. Magin and M. Ovadia, (2008) Modeling the cardiac tissue electrode interface using fractional calculus, Journal of Vibration and Control, pp 1431-1442.

[12] R. Panda and M. Dash,(2006) Fractional generalized splines and signal processing, Signal Processing, vol. 86, pp 2340-2350.

[13] S. N. Calderon-Valdez, V. F. Batlle and R. R. Perez, (2015)Fractional-order mathematical model of an irrigation main canal pool, Spanish Journal of Agricultural Research, Volume 13, Issue 3, e0212.

[14] T. T. Hartleya, C. F. Lorenzob,(2003) Fractional-order system identification based on continuous order-distributions, Signal Processing, pp 2287-2300.

[15] V. F. Batlle, A. S. Millan and R. R. Perez, (2017) Multivariable Fractional-order Model of a Laboratory Hydraulic Canal with two Pools, Proceedings of 4th International Conference on Control, Decision and Information Technologies (CoDIT'17) / April 5-7, 2017, Barcelona, Spain.

[16] V. Feliu, S. Feliu, (1997) A method of obtaining the time domain response of an equivalent circuit model, Journal of Electroanalydcal Chemistry, 435, pp 1-10.

[17] V. Feliu, J. A. Gonzalez, S. Feliu, (2004) Algorithm for extracting corrosion parameters from the response of the steel-concrete system to a current pulse, Journal of The Electrochemical Society, 151 pp 134-140.

[18] Y. Y. Tsao, B. Onaral, H. H. Sun,(1989) An algorithm for determining global parameters of minimum-phase systems with fractional power spectra, IEEE Trans. Instrum. Meas, pp 723-729.

[19] Z. Z. Yang and J. L. Zhou,(2008) An improved design for the IIR-type digital fractional order differential filter, in Proceedings of the International Seminar on Future Bio-Medical Information Engineering (FBIE 08), pp 473476.

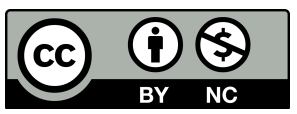

(C) 2018 by the authors. Submitted for possible open access publication under the terms and conditions of the Creative Commons Attribution CC-BYNC 3.0 license (http://creativecommons.org/licenses/bync/3.0/). 